\title{
Specimens of William Roxburgh in the Central National Herbarium at the A.J.C. Bose Indian Botanic Garden, Howrah
}

\author{
Henry John Noltie*, Anand Kumar, Kumar Avinash Bharati, Avishek Bhattacharjee and \\ Gopal Krishna
}

Sixty-one herbarium specimens collected by William Roxburgh (1751-1815) from India have recently been discovered in the Central National Herbarium (CAL), Howrah. A catalogue is provided here, including annotations of original names and determinations, currently accepted names and notes on actual or potential type status. The specimens came to CAL from eight different sources, and notes are provided on their possible origins.

Keywords: Annotations, catalogue, herbarium specimens, sources and origins, sub-collections.

WILliam ROXBURGH (1751-1815), the 'Father of Indian Botany', held the post as the first paid Superintendent of, what was then, the Calcutta Botanic Garden of the British East India Company (EIC) from 1793 to 1813 . His contribution to the study and documentation of the Indian flora was immense ${ }^{1}$, both in Calcutta and in his earlier days on the Coromandel Coast where he had started to commission artists to draw specimens, each of which was accompanied by a written description in English. He also collected large numbers of herbarium specimens that he sent to correspondents in Europe and India (see below). He seems not to have kept a 'top set' of herbarium specimens himself and for his own reference purposes relied on the drawings and descriptions (perhaps believing that dried plants would not survive in India due to the damp climate and depredations of insect larvae). The flowering plant descriptions were eventually published as Flora Indica in two posthumous editions - in 1820 and 1824 an incomplete one in two volumes by Nathaniel Wallich (Roxburgh's indirect successor at the Calcutta Botanic Garden) and William Carey, and a complete version of the phanerogams in three volumes by Carey in 1832 . Three hundred of the drawings were published under the auspices of Sir Joseph Banks as the Plants of the Coast of Coromandel (1795-1820), and a further 419 in Madras, as uncoloured lithographs, by Robert Wight between 1838 and 1845 , but the majority remained unpublished. Roxburgh's original set of drawings (the 'Roxburgh

Henry John Noltie is in the Royal Botanic Garden Edinburgh, Edinburgh EH3 5LR, United Kingdom and Anand Kumar, Kumar Avinash Bharati, Avishek Bhattacharjee and Gopal Krishna are in the Central National Herbarium, Botanical Survey of India, Howrah 711 103, India.

*For correspondence. (e-mail: HNoltie@rbge.org.uk)
Icones') is preserved at CAL; the set of contemporary facsimiles that he sent to London was lent by the EIC to the Royal Botanic Gardens, Kew (K) in 1858, for the use of Joseph Hooker, where they have remained ever since. Sanjappa et al. $^{2}$ catalogued the CAL set of Icones. The Kew collection was catalogued by $\mathrm{Sealy}^{3}$, who included notes on the wide distribution of extant Roxburgh herbarium material. Stafleu and Cowan ${ }^{4}$ gave a much fuller listing of the herbaria in which Roxburgh's specimens are now to be found, the major collections being at the Royal Botanic Garden Edinburgh (E - a set of about 600 presented to the Royal Society of Edinburgh in 1789 (ref. 5), and many hundreds more, at least some of which were probably originally sent to Daniel Rutherford and others to William Wright); the Royal Botanic Gardens, Kew ( $\mathrm{K}$ - specimens in the collections of W. J. Hooker (source unknown), George Bentham (from William Forsyth) and J. P. Rottler), with 824 in the EIC Herbarium (K-W); the Natural History Museum London (BM - originally sent to Joseph Banks and some in the collection of R. J. Shuttleworth); the National Botanic Garden of Belgium at Meise (BR - originally sent to J. E. Smith, purchased at the Linnean Society sale in 1863 by Carl Philipp von Martius -1525 of these are available on-line), and the Botanical Conservatory of the City of Geneva $(\mathrm{G}$ - originally sent to A. B. Lambert and later in the collection of Benjamin Delessert). Stafleu and Cowan ${ }^{4}$ documented smaller collections of Roxburghian specimens at Liverpool Museum (LIV - 66, perhaps South African specimens originally sent to J. E. Smith, and 68 in Royle's herbarium originally sent to J. P. Rottler); the Oxford University Botany Department (OXF); Harvard University herbaria $(\mathrm{A}$ - these are mainly fragments from other collections, including $\mathrm{BM}, \mathrm{BR}$ and $\mathrm{P}$ ); Berlin Botanical Garden (B-perhaps originally sent to Carl Ludwig 
Willdenow); Copenhagen University ( $\mathrm{C}$ - perhaps originally sent to Jens Wilken Hornemann); the National Botanic Garden, Glasnevin, Dublin (DBN); the Natural History Museum Florence (FI); New York Botanical Garden (NY); the Natural History Museum, Paris (P); Philadelphia Academy of Natural Sciences (PH); the Museum of Evolution, Uppsala (UPS - these are labelled 'e Coromandelia' so must be early collections -207 are available on-line), and the Linnean Society of London (78 of the specimens originally sent to J. E. Smith). Robinson $^{1}$ also discussed the location of Roxburgh's herbarium specimens.

It can be noticed that CAL does not appear among this list of institutions, but a small yet interesting collection of 61 Roxburgh specimens has recently been found there. These seem at some point to have been removed from the general herbarium and kept separately as a collection of historical interest. The purpose of this article is to document these specimens and reconstruct their history in so far as is possible. This effort is hampered by a lack of documentation on the formation and composition of the Calcutta herbarium, of which the only published account so far located is one by Thomas Thomson ${ }^{6}$ of the collection as it existed in 1856 , at which point the herbarium contained no Roxburgh specimens, not even a set of duplicates of the 'Wallich' (more properly EIC) Herbarium.

As Wallich was accused by some of his contemporaries of removing Roxburgh's specimens from the Calcutta Botanic Garden, it is necessary to expand here on the EIC Herbarium that he created, curated and distributed while in London between 1828 and 1832 and its relation to CAL (note 1). When he returned to Calcutta in 1832 , Wallich had intended to take one of the numerous sets of duplicates back with him but, for some unknown reason, this did not happen. It has been stated ${ }^{7}$ that Thomson, when he returned from home leave (during which he published with Joseph Hooker the first and only volume of their Flora Indica), took a set of EIC specimens, looked out by himself and Hooker, to Calcutta in 1855. Although this might have been the intention, this 'repatriation' cannot have taken place as no 'Wallich' duplicates appear in the 1856 Thomson listing $^{6}$. In fact, the first set of such specimens must have reached CAL during Thomas Anderson's superintendency, as authority to donate them was made by the Linnean Society on 5 March 1863 (note 2). It appears that some further Wallich duplicates were sent to CAL from Geneva in 1958/59 (ref. 7).

In articles published in the Calcutta Journal of Natural History in 1845, Wallich was criticized by John M'Clelland (the journal's editor and a friend of Wallich's antagonist William Griffith) and Robert Wight (for details see $\mathrm{Noltie}^{8}$ ), for what he took personally and described facetiously as 'the atrocious act I have committed in regard to the Comp[an]y's herbaria - especially
Dr Roxburgh's!' The accusation of M'Clelland and Wight was that Wallich had removed Roxburgh's specimens from the Calcutta Botanic Garden and distributed them. In fact, as will be seen, Roxburgh had not left any specimens at the Garden. As seen from the list of herbaria given above, he had sent many to Europe - to academic botanists (Rutherford in Edinburgh, Thunberg in Uppsala, Willdenow in Berlin and Hornemann in Copenhagen), to private collectors (including Banks, Smith, Lambert and apparently others, possibly also Forsyth) and to institutions (including the EIC and the Royal Society of Edinburgh); he is also known to have sent specimens to others within India, including the missionary J. P. Rottler at Tranquebar and Nathaniel Kindersley at Madras (some of Rottler's survive at LIV and K). A set of about 800 specimens sent by Roxburgh himself to the EIC in London was kept at India House, but later given to Wallich as part of his creation of the EIC Herbarium and its numerous sets of duplicates. This set appears to have contained only unicates as in a note in the "Numerical List', it is described as 'a small herbarium of the late Dr Roxburgh: no duplicates' ${ }^{\prime}$. This entire set (except perhaps for one specimen - see below) is therefore still to be found in the 'Wallich' Herbarium originally donated by the EIC to the Linnean Society of London, but transferred to Kew in $1913(\mathrm{~K}-\mathrm{W})$.

After 1832, Wallich did start a herbarium in the Calcutta Botanic Garden, including many plants collected in the Garden itself, many of which still survive (for details of early collections, see Thomson ${ }^{6}$ ). Wallich's own account of the fate of Roxburgh's own herbarium collections (and incidentally of the difficulties of preserving herbarium specimens) is to be found in a letter of selfjustification that he wrote in 1845 to Robert Wight (in response to his and M'Clelland's criticisms), of which he sent a copy to William Hooker on 8 March 1846. As this is unpublished, it is worth quoting here:

\section{Extract of a Letter from Wallich to Wight dated 2 Octo- ber 1845:}

'Your kind letter of the 10th of last month reached me on the $26^{\text {th }}$, and the following day I sent its enclosure to its destination. I do not take in the Journal of Natl. History; it has so systematically attacked me. The last number I have not even seen, though I had heard of its containing matter of that sort. That you were in any manner connected with this matter I only know from yourself, and no explanation was necessary to satisfy me, that intentionally you would never give me undeserved pain.

Are you aware that I have neither taken home, nor even sent home Dr Roxburgh's collections of dried plants? They were sent home by himself long before I came to the garden. He may have taken some, himself, when he left India in 1812 or 1813 . Wherein consists then my 
error? I did as I was commanded by the Court of Directors. Had I thought that it was desirable to reserve the specimens for being returned to this garden, assuredly would I have done my part by soliciting the Court on that score. But I was not only not blamed at the time, nor ever since, until out here, of late; but I was unanimously commended and without reservation of any sort, as far as the Company's herbariums are concerned. How is it then, that after so many years have passed I am now held up to the public in India, as having done something grievously wrong? None of the Superintendents of this Garden had left a single specimen of their botanical collections there. The fact is, it was thought that botanical specimens could only with great difficulty be preserved from decay in Bengal. I had the same idea myself for many years; and I acted upon it. I know this, that by far the completest series of Dr Roxburgh's specimens are in the Banksian herbarium; next after that in Sir J. E. Smith's and the late Mr Lambert's. I maintain that of the standard Indian collections of specimens Dr Roxburgh's is least indispensable in the garden, where is deposited his immortal volume of colored [sic] drawings made after nature. There are but very few species in Roxburgh's flora indica, or among his dried specimens which have not been accurately figured, or which cannot be satisfactorily identified by his descriptions and drawings. I do not deny that a complete series of his dried specimens would be a valuable acquisition; above all as a sacred relic of a most excellent man as well as botanist, whose memory I will revere as long as life lasts' (note 3).

As noted earlier there were no Roxburgh specimens at CAL in 1856 and this remained the case in 1895. Georgè $\mathrm{King}^{10}$, in his biographical memoir of Roxburgh, stated that 'No Indian plants of his, however, now exist in the Calcutta Herbarium'. Sixty of the 61 sheets (the South African one being an exception) must therefore have reached CAL after 1895. Nothing can be discovered about where, how or when CAL got this material, other than what can be inferred from the sheets themselves from their original labels, subsequent annotations and mounting papers (some are on 'wove' paper, which is smooth and lacks 'chain' marks; others are on 'laid' paper, which shows the lines of chain marks when held against the light). Unfortunately, the authorship of some of the handwriting is unknown and many questions remain that will only be answered if further information is found - for example, from accession details given in reports either of CAL's own or those of donating institutions. The origins turn out to be multiple, with eight different sources identified, to be discussed after an enumeration of the 61 specimens. A major donor appears to have been Kew, which is hardly surprising, as it had extensive exchange arrangements with its sister organizations over a prolonged period; but the source of the largest single 'subcollection' remains unidentified.

\section{Catalogue of Roxburgh specimens at CAL arranged in the order of his Flora Indica}

Currently accepted name (family)

Reference to description in the 1832 edition of Flora Indica; Roxburgh Icones/Description numbers at CAL and $\mathrm{K}^{2,3}$.

Name of CAL Roxburgh subcollection (explained below)

Annotations on sheets

CAL barcode

Notes (including potential type status)

1. Eranthemum pulchellum Andrews (Acanthaceae)

Fl. Ind. (1832) 1: 111, as E. pulchellum Andrews; Roxb. Icon. no. 99 (CAL \& K).

'Hb. Roxburgh', wove paper collection.

Annotations: Eranthemum pulchellum (in Roxburgh's hand); Dedalacanthus nervosus T. Anders. (in Anderson's hand); Hb Roxburgh.

CAL0000025765

\section{Graptophyllum pictum (L.) Griff. (Acanthaceae)}

Fl. Ind. (1832) 1: 117, as Justicia picta L.; Roxb. Icon. no. 908 (CAL \& K).

'Hb. Roxburgh', wove paper collection.

Annotations: Justicia picta (in Roxburgh's hand); $\mathrm{Hb}$

Roxburgh.

CAL0000025794

3. Justicia adhatoda L. (Acanthaceae)

Fl. Ind. (1832) 1: 126, as J. Adhatoda Willd.; Roxb. Icon. no. 1201 (CAL \& K).

'Hb. Roxburgh', wove paper collection.

Annotations: Justicia adhatoda (in Roxburgh's hand); $\mathrm{Hb}$ Roxburgh.

CAL0000025754

Note. On the same sheet are two specimens annotated by Wallich 'E Nepalia, 1819' (these must have been collected by Edward Gardner) that also have a printed 'Herb T. Anderson M.D.' label annotated by Anderson 'Adhatoda vasica, Nepal $2560 \mathrm{ft}$, Wallich'. This suggests that the composite sheet was made up by Anderson (possibly at CAL 1861-68).

4. Nicoteba betonica (L.) Lindau (Acanthaceae)

Fl. Ind. (1832) 1: 128, as Justicia betonica L.; Roxb. Icon. no. 1769 (CAL \& K).

'Peninsula Indiae Orientalis', laid paper collection.

Annotations: Justicia betonica Linn., Peninsulae Indiae Orientalis (unknown hand).

CAL0000025783

5. Nelsonia canescens (Lam.) Spreng. (Acanthaceae)

Fl. Ind. (1832) 1: 131, as Justicia tomentosa Roxb.; Roxb. Icon. no. 519 (CAL \& K).

'Peninsula Indiae Orientalis', laid paper collection. 
Annotations: Justicia tomentosa, Peninsulae Indiae Orientalis, Roxburgh (unknown hand); Nelsonia tomentosa Willd. (unknown hand).

\section{CAL0000025781}

Note. Potential original material of Justicia tomentosa Roxb. (an illegitimate later homonym of $J$. tomentosa Vahl 1804?).

6. Justicia glauca Rottler (Acanthaceae)

Fl. Ind. (1832) 1: 132, as J. orixensis Kön Mss.; Roxb. Icon. s.n.

'Peninsula Indiae Orientalis', laid paper collection.

Annotations: Dianthera arvensis, Peninsulae Indiae Orientalis, Roxburgh (unknown hand); Justicia orixensis Roxb. (unknown hand).

\section{CAL0000025785}

Note: Impossible to know if this is the original material of $J$. orixensis, as the name is in a later hand.

7. Rostellularia quinqueangularis (J. König ex Roxb.) Nees (Acanthaceae)

(Misidentified by ?Roxburgh as Justicia repens).

'Peninsula Indiae Orientalis', laid paper collection.

Annotations: Justicia repens, Peninsulae Indiae Orientalis, Roxburgh (unknown hand); Justicia (Rostellaria) peploides T. And. and peploides T. And. (in Anderson's hand).

CAL0000025788

8. Rostellularia diffusa (Willd.) Nees (Acanthaceae) Fl. Ind. (1832) 1: 132, as Justicia procumbens L.; Roxb. Icon. no. 515 (CAL \& K).

'Hb. Roxburgh', wove paper collection.

Annotations: Justicia procumbens (in Roxburgh's hand);

Rostellularia diffusa $\mathrm{N}$ ab Esen (in Anderson's hand); $\mathrm{Hb}$

Roxburgh.

CAL0000025782

Note. The name $J$. procumbens was not published.

9. Rungia pectinata (L.) Nees (Acanthaceae)

F1. Ind. (1832) 1: 133, as Justicia pectinata Roxb.; Roxb. Icon. no. 517 (CAL \& K).

'Hb. Roxburgh', wove paper collection.

Annotations: Justicia pectinata (in Roxburgh's hand);

Rungia pectinata $\mathrm{N}$ ab E (in unknown hand); Hb Roxburgh.

\section{CAL0000025768}

10. Cyperus difformis L. (Cyperaceae)

Fl. Ind. (1832) 1: 195, as C. difformis L.; Roxb. Icon. 716 (CAL \& K).

Herb. Lambert, ex Geneva collection.

Annotations: Ind: Orien: Dr. Roxburgh (unknown 18th century hand); Cyperus difformis Linn. (determination by C.B. Clarke, 3 April 1895).

CAL0000025758
11. Fimbristylis alboviridis C.B.Clarke (Cyperaceae)

(No original determination to species).

'Peninsula Indiae Orientalis', laid paper collection.

Annotations: Scirpus, Peninsulae Indiae Orientalis, Roxburgh (unknown hand); Fimb. Albo-viridis n. sp., C.B. Clarke m.s. (determination by Clarke, Jan 1884 - but not a syntype).

CAL0000025760

12. Fimbristylis pubisquama J. Kern (Cyperaceae)

(The original determination is erroneous).

'Peninsula Indiae Orientalis', laid paper collection.

Annotations: Cyperus fuscus, Pen. Ind. Orient., Roxburgh (unknown hand); Fimbristylis compress a Boeck. Determination by C.B. Clarke, Dec 1883); Nut fuscous, smooth ([=] Cyperus fuscus [sensu] Roxb.) (determination by Clarke)

\section{CAL0000001760}

13. Fimbristylis ferruginea (L.) Vahl (Cyperaceae)

(Scirpus campestrisis not treated in Fl. Ind.).

?'Peninsula Indiae Orientalis', laid paper collection.

Annotations: Scirpus campestris (in Roxburgh's hand).

Two old tickets with 'Roxburgh' in unknown old hand and annotated Fimbristylis ferruginea Vahl by C.B. Clarke, one in Feb 1885, the other Feb 1888.

CAL0000001808

Note: The name $S$. campestris was not published.

14. Miscanthus fuscus (Roxb.) Benth. (Gramineae)

Fl. Ind. (1832) 1: 241, as Saccharum fuscum Roxb.; Roxb. Icon. 1114 (CAL \& K).

Forsyth/Bentham, laid paper collection.

Annotations: East India Dr Roxburgh, Forsyth, Purch. 1835 (printed label); Herbarium Benthamianum 1854 (ink stamp); Saccharum fuscum Roxb. (pencil annotation in unknown hand).

CAL0000001808

Note: Potential original material of S. fuscum Roxb.

15. Paspalum scrobiculatum L. (Gramineae)

Fl. Ind. (1832) 1: 278, as P. scrobiculatum L.; Roxb. Icon. 777 (CAL \& K).

Forsyth/Bentham, laid paper collection.

Annotations: East India Dr Roxburgh, Forsyth, Purch. 1835 (printed label); Herbarium Benthamianum 1854 (ink stamp); P. scrobiculatum Linn. (determination by C.E.C. Fischer for Flora of Madras, 17 iv 1931).

CAL0000025761

16. Digitaria bicornis (Lam.) Roem. \& Schult. (Gramineae)

Forsyth/Bentham, laid paper collection.

Annotations: East India Dr Roxburgh, Forsyth, Purch. 1835 (printed label); Herbarium Benthamianum 1854 (ink 
stamp); $\mathrm{P}$ [anicum] sanguinale Lamk. var. commutatum (determination in Bentham's hand).

CAL0000025759

Note: A Jenkins specimen from Assam determined by Bentham as 'var. extensa' is mounted on the same sheet.

17. Aristida hystrix L.f. (Gramineae)

Fl. Ind. (1832) 1: 350, as A. hystrix L.; Roxb. Icon. 856 (K).

W.J. Hooker/'Hb Roxburgh', wove paper collection.

Annotations: A. Hystrix L. (possibly in Wallich's hand); $\mathrm{Hb}$ Roxburgh (unknown hand); Herbarium Hookerianum 1867 (ink stamp).

CAL0000025779

18. Oropetium thomaeum (L.f.) Trin. (Gramineae)

Fl. Ind. (1832) 1: 357, as Rottboellia Thomaea L.; Roxb. Icon. 866 (CAL \& K).

'Peninsula Indiae Orientalis', laid paper collection.

Annotations: Rottboellia pygmaea, Peninsulae Indiae Orientalis, Roxburgh (unknown hand).

CAL0000025773

19. Glinus oppositifolius (L.) Aug. DC. (Molluginaceae) (Not treated under Mollugo spergula by Roxburgh).

'Peninsula Indiae Orientalis', laid paper collection.

Annotations: Mollugo spergula Linn., Pharnaceum mollugo Lam., Pen. Indiae Orientalis, Roxburgh (unknown hand).

CAL0000025776

20. Santalum album L. (Santalaceae)

Fl. Ind. (1832) 1: 442, as S. album L.; Roxb. Icon. 1944 (CAL \& K).

?'Peninsula Indiae Orientalis', laid paper collection.

Annotations: Santalaceae, Santalum, Roxburgh (unknown hand).

CAL0000024824

21. Merremia emarginata (Burm.f.) Hallier f. (Convolvulaceae)

(Original identification only to genus).

'Peninsula Indiae Orientalis', laid paper collection.

Annotations: Convolvulus, Pen. Ind. Orient., Roxburgh (unknown hand); Ipomoea reniformis Choisy (determination in a later unknown hand).

CAL0000025766

Note: Watermark - Sevans \& Co 1851.

22. Spermadictyon suaveolens Roxb. (Rubiaceae)

Fl. Ind. (1832) 1: 554, as Hamiltonia suaveolens Roxb.;

Roxb. Icon. no. 1364 (CAL).

?'Hb. Roxburgh', wove paper collection.

Annotations: Hamiltonia suaveolens (in unknown early hand); Dr Roxburgh (unknown hand).

CAL0000024831

Note: Potentially original material of $S$. suaveolens.
23. Cordia sp. (Boraginaceae)

(Not treated under Cordia buxifolia (a West Indian species) by Roxburgh).

?'Peninsula Indiae Orientalis', laid paper collection.

Annotations: Cordia buxifolia, East India, Roxburgh (unknown hand); Cordiaceae (unknown later hand in pencil).

CAL0000025751

\section{Rhamnus virgata Roxb. (Rhamnaceae)}

(No original name $-R$. hirsuta dates from 1834. Fl. Ind. (1832) 1: 604, as R. virgatus Roxb.; Roxb. Icon. no. 2450 (CAL), $2448(\mathrm{~K}))$.

?'Peninsula Indiae Orientalis', laid paper collection.

Annotations: Rhamnus hirsuta W \& A, India, Roxburgh (unknown hand).

CAL0000024828

25. Ziziphus lotus (L.) Lam. (Rhamnaceae)

Fl. Ind. (1832) 1: 610, as Z. lotus Lam.; Roxb. Icon. s.n.

?'Peninsula Indiae Orientalis', laid paper collection.

Annotations: Rhamnus Lotus? India. Are these two the same plants, Roxburgh (unknown hand).

CAL0000024827

26. Ziziphus oenoplia (L.) Mill. (Rhamnaceae)

F1. Ind. (1832) 1: 611, as Z. oenoplia Willd.; Roxb. Icon. s.n.

?'Peninsula Indiae Orientalis’, laid paper collection.

Annotations: Ziziphus oenoplia, India, Roxburgh (unknown hand).

CAL0000024826

27. Ziziphus xylopyrus (Retz.) Willd. (Rhamnaceae)

(No original name, but Fl. Ind. (1832) 1: 611, as Z. xylopyrus Willd.; Roxb. Icon. no. 579 (CAL \& K)).

'Peninsula Indiae Orientalis', laid paper collection.

Annotations: Rhamneae, Pen. Ind. Orient., Roxburgh (unknown hand); Ziziphus xylopyrus Willd. (unknown later hand); a Roxburghian specimen (unknown later hand).

CAL0000025793

28. Gymnosporia emarginata (Willd.) Thwaites (Celastraceae)

(No original name, but Fl. Ind. (1832) 1: 620, as Celastrus emarginatus Willd; Roxb. Icon. 593 (CAL \& K)).

?'Peninsula Indiae Orientalis', laid paper collection.

Annotations: Rhamnaceae, Celastrineae, Roxburgh (unknown hand).

CAL0000024836

29. Gardenia resinifera Roth (Rubiaceae)

Fl. Ind. (1832) 1: 707, as G. lucida Roxb.; Roxb. Icon. 1543 (CAL), 1542 (K).

Roxburgh in Herb. EIC collection.

Annotations: Gardenia lucida (in Wallich's hand); $\mathrm{Hb}$ Roxburgh (Wall. Cat. no.) 8270a (in unknown hand); 
'Wallich Sheet' (printed label); Gardenia resinifera, Type of G. lucida Roxb. D.D. Tirvengadum 4111974 (determination by Tirvengadum).

\section{CAL0000016087}

Note: Possible syntype, lectotype, or isolectotype of $G$. lucida.

30. Centella asiatica (L.) Urb. (Umbelliferae)

Fl. Ind. (1832) 2: 88, as Hydrocotyle asiatica Willd.; Roxb. Icon. 1385 (CAL \& K).

?'Peninsula Indiae Orientalis', laid paper collection.

Annotations: Hydrocotyle Asiatica, India, Roxburgh (unknown hand).

CAL0000025749

31. Sanicula elata Buch.-Ham. ex D.Don (Umbelliferae) (Not treated under Sanicula elata in Fl. Ind.).

'Peninsula Indiae Orientalis', laid paper collection.

Annotations: Sanicula elata Ham., Peninsula Indiae Orient., Roxburgh (unknown hand).

CAL0000024833

32. Pisonia aculeata L. (Nyctaginaceae)

Fl. Ind. (1832) 2: 217, as P. aculeata Willd.; Roxb. Icon. no. 481 (CAL \& K).

'Peninsula Indiae Orientalis', laid paper collection.

Annotations: Pisonia, Peninsula Indiae Orient., Roxburgh (unknown hand).

CAL0000024834

33. Memecylon umbellatum Burm.f. (Melastomataceae) (Not treated under M. tinctorium in Fl. Ind.).

'Peninsula Indiae Orientalis', laid paper collection.

Annotations: Memecylon tinctorium Koen., ex Peninsula Indiae Orient., Roxburgh (unknown hand).

CAL0000025763

34. Polygonum plebeium R.Br. (Polygonaceae)

( $P$. divaricatum not treated in Fl. Ind.).

'Peninsula Indiae Orientalis', laid paper collection.

Annotations: Polygonum divaricatum, Peninsula Indiae Orientalis, Roxburgh (unknown hand); Polygonum plebejum Br. (later determination in unknown hand).

CAL0000025786

Note: The name $P$. divaricatumwas not published.

35. Pterolobium hexapetalum (Roth) Santapau \& Wagh (Leguminosae)

Fl. Ind. (1832) 2: 367, as Caesalpinia lacerans Roxb.; Roxb. Icon. 60 (CAL).

?'Peninsula Indiae Orientalis', laid paper collection.

Annotations: Pterolobium lacerans, Roxburgh (unknown hand); Pterolobium indicum Rich. (not P. lacerans Br.; later determination in unknown hand).

CAL0000025750

Note: Possible original material of C. lacerans Roxb.
36. Mespilus japonica Thunb. (Rosaceae)

Fl. Ind. (1832) 2: 510, as M. japonica Banks; Roxb. Icon. no. 948 (CAL \& K).

'Hb. Roxburgh', wove paper collection.

Annotations: Ticket 1: Mespilus pomifera 666 (in Roxburgh's hand), 'pomifera' scored out and replaced by 'japonica' in a slightly later hand. Ticket 2: 666 Roxb. ticket (unknown 18th century hand).

CAL0000025778

Note: Numbers on Roxburgh specimens are extremely rare, and it is not known to what this one refers.

37. Diospyros chloroxylon Roxb. (Ebenaceae)

(The name Jambolifera pedunculata is not treated in Fl. Ind.).

?'Peninsula Indiae Orientalis', laid paper collection.

Annotations: Ticket 1: Jambolifera pedunculata (Roxburgh's hand); Roxburgh (unknown hand). Ticket 2: Jambolifera pedunculata. Peninsula Indiae Orientalis, Roxburgh, Ebenaceae (unknown hand); Diospyros chloroxylon Roxb. 'J.B.' (meaning unknown; later determination in unknown hand).

CAL0000025756

38. Crateva religiosa G.Forst. (Capparaceae)

Fl. Ind. (1832) 2: 571, as Crateva trifoliata Roxb.; Roxb. Icon. no. 157 (CAL \& K).

'Hb. Roxburgh', wove paper collection.

Annotations: Capparis trifoliata (?Roxburgh's hand); $\mathrm{Hb}$ Roxburgh (pencil); Crataeva Roxburghii (determination in unknown hand - deleted and replaced with C. Nurvala Ham.)

CAL0000033340

Note: Possibly original material of Capparis trifoliata Roxb.

39. Grewia asiatica L. (Malvaceae)

Fl. Ind. (1832) 2: 586, as G. asiatica Willd.; Roxb. Icon. 223 (CAL \& K).

'Peninsula Indiae Orientalis', laid paper collection.

Annotations: Grewia Asiatica, Peninsula Indiae Orientalis, Roxburgh (unknown hand); Grewia tiliaefolia Vahl (old redetermination in unknown hand); Grewia tiliifolia Vahl (determination by V. Narayanaswami 16. 5 . 1944).

\section{CAL0000025772}

40. Grewia asiatica L. (Malvaceae)

Fl. Ind. (1832) 2: 586, as G. asiatica Willd.; Roxb. Icon. 223 (CAL \& K).

?'Peninsula Indiae Orientalis', laid paper collection.

Annotations: Grewia Asiatica, Roxburgh (unknown hand); Grewia tiliifolia Vahl (determination by $\mathrm{V}$. Narayanaswami 16. 5. 1944).

CAL0000025943 
41. Microcos paniculata L. (Malvaceae)

(G. microcos is not treated in Fl. Ind.).

'Peninsula Indiae Orientalis', laid paper collection.

Annotations: Grewia microcos Linn., Peninsula Indiae

Orientalis, Roxburgh (unknown hand); Grewia bracteata

Heyne ex Roth (determination by V. Narayanswami 18.5.1944).

CAL0000025775

42. Acanthus ilicifolius L. (Acanthaceae)

Fl. Ind. (1832) 3: 32, as A. ilicifolius Willd.; Roxb. Icon. no. 966 (CAL \& K).

'Hb. Roxburgh', wove paper collection.

Annotations: Acanthus ilicifolius (in Roxburgh's hand);

Hb Roxburgh.

CAL0000025767

Note: On the same sheet is mounted a J.S. Law specimen labelled 'North \& South Concan'.

43. Barleria prionitis L. (Acanthaceae)

Fl. Ind. (1832) 3: 36, as B. prionites Willd.; Roxb. Icon. no. 331 (CAL \& K).

'Hb. Roxburgh', wove paper collection.

Annotations: Ticket 1: Barleria prionitis (in Roxburgh's hand); Hb Roxburgh; later annotation Barleria Prionitis Linn., specimen from Roxburgh's Herb. Ticket 2: Barleria Prionitis Linn., Roxburgh (unknown hand).

CAL0000025777

44. Crossandra infundibuliformis (L.) Nees (Acanthaceae)

Fl. Ind. (1832) 3: 41, as Ruellia infundibuliformis Roxb.; Roxb. Icon. no. 97 (CAL \& K).

'Hb. Roxburgh', wove paper collection.

Annotations: Ruellia infundibuliformis Justicia Linn. (in Roxburgh's hand); Hb Roxburgh.

CAL0000025770

45. Asystasia gangetica (L.) T. Anderson (Acanthaceae) Fl. Ind. (1832) 3: 42, as R. Zeylanica Kön. Mss.; Roxb. Icon. no. 1987 (CAL \& K).

'Hb. Roxburgh', wove paper collection.

Annotations: Ruellia secunda Buch. (unknown old hand, but not Roxburgh's); Hb Roxburgh; Asystysia gangetica T. And. (in Anderson's hand).

CAL0000025784

46. Phaulopsis dorsiflora (Retz.) Santapau (Acanthaceae)

Fl. Ind. (1832) 3: 48, as Ruellia imbricata Willd.; Roxb. Ic. No. 1470 (CAL \& K).

'Hb. Roxburgh', wove paper collection.

Annotations: Ruellia glutinosa (in Roxburgh's hand); $\mathrm{Hb}$

Roxburgh; Phylopsis (sic) parviflora Willd. (later determination, perhaps in Anderson's hand?).

CAL0000025791
47. Hygrophila erecta (Burm.f.) Hochr. (Acanthaceae)

(Not treated under Barleria inermis in Fl. Ind.).

?'Hb. Roxburgh', wove paper collection.

Annotations: Hygrophila quadrivalvis T. Anderson forma typica N ab Esen., Barleria inermis Roxb., Roxburgh's specimen (in Anderson's hand).

CAL0000025769

Note: The name $B$. inermis was not published.

48. Ruellia crispa L. (Acanthaceae)

( $R$. crispa is not treated in Fl. Ind.; an earlier misidentification on Roxburgh's part).

'Hb. Roxburgh', wove paper collection.

Annotations: Ticket 1: Ruellia crispa, blue flowers (in Roxburgh's hand); Hb Roxburgh. Ticket 2: Herb T. Anderson MD, Hemigraphis Dura T. Anders., Ruellia Dura $\mathrm{N}$ ab Esen. in herb. Wight, Roxburgh (Anderson's printed label filled out in his own hand).

CAL0000025787

49. Dalbergia volubilis Roxb. (Leguminosae)

Fl. Ind. (1832) 3: 231, as D. volubilis Roxb.; Roxb. Icon. 83 (CAL \& K).

'Peninsula Indiae Orientalis', laid paper collection.

Annotations: Dalbergia frondosa Roxb. Pen. Ind. Orient. (unknown hand); Dalbergia volubilis Roxb. (later determination, replacement for scored out 'frondosa' in unknown hand). This is a Roxburghian sheet (illeg initials; later pencil annotation in unknown hand).

CAL0000024830

Note: Impossible to confirm if it is original material as the name $D$. volubilis is in a later hand.

50. Butea superba Roxb. ex Willd. (Leguminosae)

Fl. Ind. (1832) 3: 247, as B. superba Willd.; Roxb. Icon. 68 (CAL \& K).

?'Peninsula Indiae Orientalis', laid paper collection.

Annotations: Butea scandens Roxburgh (unknown hand); Butea superba Roxb. (later determination in unknown hand).

CAL0000025942

51. Indigofera subulata Poir. var. scabra (Roth) Meikle (Leguminosae)

Fl. Ind. (1832) 3: 375, as I. flaccida Kön. Mss.; Roxb. Icon. 384 (CAL \& K).

?'Peninsula Indiae Orientalis’, laid paper collection.

Annotations: Indigofera flaccida, Cakondah, India, Roxburgh (unknown hand); I. subulata Vahl, (determination by J.S. Gamble for Flora of Madras, 1916); I. trita L. f. var. scabra (Roth) Ali=I. scabra Roth, Type of I. flaccida Koen. ex Roxb (annotation by M. Sanjappa 15.6.1982).

CAL0000025764

Note: Localities on Roxburgh specimens are exceptionally rare - Corkonda $=$ Korukonda $(6 \cos =\mathrm{c} 13$ miles north 
of Rajamundry) is where Roxburgh leased a farm for 5 years in June 1793 (just as he was leaving for Calcutta). Note: Impossible to confirm if this is original material as the name I. flaccida is in a later hand.

\section{Tephrosia tinctoria (L.) Pers. (Leguminosae)}

Fl. Ind. (1832) 3: 386, as Galega tinctoria Willd.; Roxb. Icon. s.n.

'Peninsula Indiae Orientalis', laid paper collection.

Annotations: Galega tinctoria, Pen. Indi. Orient., Leguminosae, Roxburgh (unknown hand); Tephrosia purpurea Pers. var. maxima Baker (later annotation in unknown hand); Teph. maxima Pers. (determination by J.S. Gamble for Flora of Madras, 1917).

\section{CAL0000025752}

53. Ficus assamica Miq. (Moraceae)

Fl. Ind. (1832) 3: 555, as F. repens Willd; Roxb. Icon. 1902 (CAL), 1099 (K).

W.J. Hooker/'Hb Roxburgh', wove paper collection. Mounted on same sheet as 54 .

Annotations: Ficus repens (Roxburgh's hand), Roxburgh (unknown hand); Herbarium Hookerianum 1867 (ink stamp); F. heterophylla var. repens Willd. (determination by D. ?Bam, 2311 1995); Ficus assamica Miq. 'Roxburgh collection may be type' (determination by J.V. Sudhakar 18. 5. 2012).

CAL0000025720

Note: The name F. repens Rottler was based on Rottler specimens; so this is unlikely to be a type.

\section{Ficus exasperata Vahl (Moraceae)}

Fl. Ind. (1832) 3: 555, as F. exasperata Roxb.; Roxb. Icon. 1751 (CAL), $1734(\mathrm{~K})$.

W.J. Hooker/'Hb Roxburgh', wove paper collection. Mounted on same sheet as 53 .

Annotations: Ficus exasperata (in ?Roxburgh's hand), $\mathrm{Hb}$ Roxburgh; Herbarium Hookerianum 1867 (ink stamp). This is a distinct species from Amboyna see Roxb. Fl. iii 555, W.B. H(emsley) (annotation made at Kew by Hemsley).

CAL0000025780

55. Scleria corymbosa Roxb. (Cyperaceae)

Fl. Ind. (1832) 3: 574, as $S$. corymbosa Roxb. (the name Carex orixensisis not treated); Roxb. Icon. s.n.

W.J. Hooker/'Hb Roxburgh', wove paper collection. Mounted on same sheet as 56.

Annotations: Carex orixensis (Roxburgh's hand), $\mathrm{Hb}$ Roxburgh (unknown hand); Herbarium Hookerianum 1867 (ink stamp); Scleria corymbosa Roxb. (determination in C.B. Clarke's hand).

CAL0000002931

Note: Annotated as isotype of Scleria corymbosa Roxb. by Sangita Dey, 8. 9. 2008.
56. Scleria corymbosa Roxb. (Cyperaceae) Fl. Ind. (1832) 3: 574, as S. corymbosa Roxb.; Roxb. Icon. s.n.

'Hb. Roxburgh', wove paper collection. Mounted on same sheet as 55 .

Annotations: S. androgyna Nees ([Roxburgh's] name lost; in Anderson's hand), Hb Roxburgh; Scl. corymbosa Roxb. (determination in C.B. Clarke's hand).

CAL0000002932

Note: Annotated as isotype of Scleria corymbosa Roxb. by Sangita Dey, 8. 9. 2008.

A third specimen mounted on the same sheet, from Silhet, determined as Scleria elata by C.B. Clarke.

57. Pipturus argenteus (G.Forst.) Wedd. (Moraceae)

Fl. Ind. (1832) 3: 599, as Morus paniculata Roxb.; Roxb. Icon. 2401 (CAL), 1675 (K).

W.J. Hooker/'Hb Roxburgh', wove paper collection.

Annotations: [Male symbol] Morus paniculata (Roxburgh's hand), Hb Roxburgh (unknown hand); Herbarium Hookerianum 1867 (ink stamp); Pipturus velutinus Wedd. (determination in J.D. Hooker's hand).

CAL0000025789

Note: Possible original material of Morus paniculata Roxb.

58. Viscum orientale Willd. (Santalaceae)

Fl. Ind. (1832) 3: 764, as V. verticillatum Willd.; Roxb. Icon. s.n.

'Peninsula Indiae Orientalis', laid paper collection.

Annotations: Viscum verticillatum, Pen. Ind. Orient., Roxburgh (unknown hand); V. orientale Willd. (vide F.B.I; later determination in unknown hand, but probably made at Kew).

CAL0000025753

59. Suregada multiflora (A.Juss.) Baill. (Euphorbiaceae)

(Not treated in Fl. Ind.).

'Peninsula Indiae Orientalis', laid paper collection.

Annotations: Euphorbiaceae, Pen. Ind. Orient., Roxb. (unknown hand); Gelonium multiflorum A. Juss. (later determination by N.P. Balakrishnan, date?).

CAL0000024829

60. Juniperus semiglobosa Regel (Cupressaceae)

(No original name).

Botanical Society of Edinburgh collection.

Annotations: Societas Botanica Edinensis, Tamarix dioica Roxb., India, by Roxburgh (printed label annotated with name in unknown hand); Juniper (unknown hand); J. pseudosabina Fisch. \& Mey. (later determination in unknown hand).

CAL0000025757

Note: The determination is taken from a similar specimen at E made by A. Farjon (17 July 2000).

CURRENT SCIENCE, VOL. 120, NO. 6, 25 MARCH 2021 
61. Erica $\mathrm{sp}$. (Ericaceae)

(No original name).

Sibella Roxburgh collection.

CAL0000027002

\section{Notes on possible origins of the 'subcollections'}

\section{Subcollections certainly originating from Kew}

1. Ex Herb. George Bentham: Three sheets (nos 14, 15 and 16; all grasses), mounted on laid paper with the ink stamp 'Herbarium Benthamianum 1854'. These specimens were purchased by Bentham from the collection of William Forsyth junior (1772-1835) and are labelled merely 'East India Dr Roxburgh'. Forsyth was a partner in a London nursery, but owned a well-known library. So he could have acquired the Roxburgh specimens himself, but it is also possible that they came to him through his father William Forsyth senior (1737-1804), who had been curator of the Chelsea Physic Garden and later of the royal palaces in London. Bentham's herbarium was given to Kew in 1854 , but these sheets must have been sent with the Hooker sheets by $\mathrm{K}$ to $\mathrm{CAL}$ much later, perhaps with the 'Hb. Roxburgh' collection.

2. Ex Herb. Sir William Jackson Hooker: Five sheets (nos 17, 53, 54, 55 and 57), mounted on wove paper with the ink stamp 'Herbarium Hookerianum 1867' (the date of purchase of the collection by K). Of these, four bear the plant name in Roxburgh's hand (the other is possibly in Wallich's) and four are annotated ' $\mathrm{Hb}$ Roxburgh', making a significant link with the much larger ' $\mathrm{Hb}$ Roxburgh' collection (no. 3 below). It is not known how or when Hooker obtained his Roxburgh specimens (and there is no mention of them in the account of his Indian collections by J.D. Hooker and Thomson ${ }^{11}$, but it cannot have been from Roxburgh himself. Like the Bentham collection, these sheets must have been sent to CAL as duplicates from $\mathrm{K}$, but at an unknown date.

\section{Possibly originating from Kew}

3. The 'Hb Roxburgh' set mounted on wove paper: Sixteen sheets - the second largest of the subcollections (nos 1-3, 8, 9, 22, 37, 38, 42-48 and 56). These have slitted tickets (showing that the specimen was originally inserted into the label) with the name in Roxburgh's own hand and a later annotation 'Hb Roxburgh', and are mounted on wove paper. The format of the ticket is the same as for the Roxburgh specimens in Hooker's herbarium, which suggests that they came from the same source. No fewer than 12 of these are Acanthaceae and many are annotated by Thomas Anderson who specia- lized in the family, but from King's ${ }^{10} 1895$ statement they cannot have reached CAL during Anderson's superintendency of the Herbarium (from 1861 to 1868). These might have been duplicates that were at Kew but not incorporated into Hooker's herbarium there, annotated by Anderson when he worked at $\mathrm{K}$ and later sent to CAL.

\section{From the EIC Herbarium}

4. Of the 842 Roxburgh sheets in the EIC ('Wallich') Herbarium (searchable on the Royal Botanic Garden Edinburgh's Wallich website), there is a single one at CAL (no. 29, Gardenia resinifera). According to Wallich's ${ }^{9}$ list, there were no duplicates of the Roxburgh sheets. These specimens had been sent by Roxburgh himself to the India Museum before 1813, but in 1829 were given along with other Indian museum herbaria (of Russell, Wight and 'Hb. Madras'), to Wallich for distribution. Wallich himself failed to take back a set of EIC duplicates when he returned to Calcutta in 1832, and it was not until the 1860 s that the Linnean Society sent CAL its set of duplicates (see introduction above).

\section{Possibly from the Royal Botanic Garden Edinburgh}

5. A single specimen (no. 60, Juniperus subglobosa) has a printed Botanical Society of Edinburgh (BSE) label. One of the original aims of the BSE in 1836 was to form an herbarium, and there were close links between the Society and the University of Edinburgh, which had its own herbarium. The two collections were united in 1838 and later became the herbarium of RBGE. Most of the Roxburgh specimens at E were probably already in the University herbarium, but perhaps some duplicates were given to the BSE for distribution (there is at least one BSE/Roxburgh specimen at E). It is impossible to say how this came to Calcutta, but one possibility is that it was also through Thomas Anderson, a BSE member who, with Hugh Cleghorn in 1849/1850, recurated the Indian elements of the University Herbrarium.

\section{Of unknown provenance}

6. The 'Peninsula Indiae Orientalis' collection: With 33 sheets this is the largest but most puzzling of the subcollections and covers a wide range of families (nos 4-7, $11-13,18-21,23-28,30-36,39-41,49-52$ and 58-59). None of them bears Roxburgh's writing and nearly all of the tickets are annotated 'Peninsula Indiae Orientalis' (variously abbreviated) in a later hand. In Roxburgh's time the usual geographical term used was 'East Indies' or 'India', and 'Peninsula Indiae Orientalis' suggests that the tickets are post-1834 when the term was used by Wight and Arnott. The specimens must have been in an 
unknown, post-Roxburghian collection and later (one has an 1851 watermark) mounted, surely in the UK, on a high-quality laid paper. None, at least of those digitized, of the Roxburgh sheets at $\mathrm{K}$ has this type of label, but some of the sheets in this subcollection are annotated by C.B. Clarke (1883, 1884, 1885, 1888 and 1895), J.S. Gamble (in 1916 and 1917) and C.E.C. Fischer (1931), all of whom did work at Kew in their post-Indian days. This does not necessarily mean that they originated from Kew, as the sheets could have been lent for study to $\mathrm{K}$ either by CAL or by some other herbarium. The only way their origin and date of donation to CAL is likely to be solved is if sheets with the same Peninsula Indiae Orientalis ticket are found on specimens in another herbarium (and it should be noted that none at $\mathrm{E}$ bears this type of label).

\section{From Geneva}

7. A single specimen (no. 10, Cyperus difformis), which must have been sent by Roxburgh to Aylmer Bourke Lambert. At the sale of Lambert's herbarium in 1833, the large set (2000-2250 plants) of specimens sent by Roxburgh to Lambert was purchased by Obadiah Rich on behalf of the French botanist Benjamin Delessert, and have ended up in Geneva $(G)^{12}$. This specimen was sent as a duplicate to CAL from the Conservatorio Botanico Genevensis at an unknown date.

\section{Donated by Sibella Roxburgh}

8. A single specimen (no. 61, Erica sp.), of a South African heath, with a specially printed label: 'HERB. HORT. BOT. CALCUTTENSIS. Collected at the Cape of Good Hope in the year 1797 by Dr. W. ROXBURGH, Second Superintendent of the Botanical Garden, Calcutta. Presented to the Herbarium of this garden in June 1877 by his daughter, Miss ROXBURGH. G. KING, Superintendent'.

Only one such specimen has been found so far, but there must be others as King ${ }^{10}$ wrote of 'a number of plants, collected at the Cape of Good Hope during his last voyage to England [this is incorrect; on his final voyage he stopped at St Helena rather than the Cape]'. Coincidentally, Sibella was born at sea off the Cape where she was baptized in 1807, a decade after her father's 18- month sick leave spent there (he had arrived there in April 1798, rather than as stated on the label as 1797).

\section{Notes}

1. Fraser-Jenkins ${ }^{7}$ also gives a useful list of the 66 institutions in Europe and Russia to which he sent sets (many partial) of the specimens that he took from CAL to London in 1828 .

2. Council Minute Book of Linnean Society of London for 5 March 1863 (M.F. Watson, pers. commun.).

3. Volume of letters, Wallich to W.J. Hooker, 1841-49, Royal Botanic Gardens, Kew, UK.

1. Robinson, T. F., William Roxburgh (1751-1815): the Founding Father of Indian Botany, Phillimore in association with the Royal Botanic Garden Edinburgh, Chichester, UK, 2008.

2. Sanjappa, M., Thothathri, K. and Das, A. R., Bull. Bot. Surv. India, 1993, 33, 1-232.

3. Sealy, J. R., Kew Bull., 1956, 297-399.

4. Stafleu, F. A. and Cowan, R. S., Taxonomic Literature - II, Bohn, Scheltema \& Holkema, Utrecht, The Netherlands, 1983, vol. 4, p. 983.

5. Waterston, C. D., Collections in Context, National Museums of Scotland, Edinburgh, UK, 1997.

6. Thomson, T., Hooker's J. Bot. Kew Gard. Misc., 1857, 9, 10-4; 33-41 (reprinted from J. Asiatic Soc. Bengal, 25(5), 405-118).

7. Fraser-Jenkins, C. R., The First Botanical Collectors in Nepal: the fern collections of Hamilton, Gardner and Wallich, Bishen Singh Mahendra Pal Singh, Dehra Dun, 2006, p. 49; 58.

8. Noltie, H. J., The Life and Work of Robert Wight, Royal Botanic Garden Edinburgh, UK, 2007, p. 156.

9. Wallich, N., A Numerical List of Dried Specimens of Plants in the East India Company's Museum, Collected Under the Superintendence of Dr Wallich of the Company's Botanic Garden at Calcutta, Honorable East India Company, London, UK, 1828-49, p. 60.

10. King, G., Ann. R. Bot. Gard. Calcutta, 1895, 5, 1-9.

11. Hooker, J. D. and Thomson, T., Flora Indica, Vol. 1, W. Pamplin, London, UK, 1855, p. 65.

12. Miller, H. S., Taxon, 19, 489-533.

ACKNOWLEDGEMENTS. H.J.N. thanks Dr A. A. Mao (Director, Botanical Survey of India (BSI), Kolkata) and Dr V. P. Prasad (Head of Office, Central National Herbarium (CNH), Howrah) for permission to consult the Roxburgh collections on a visit to CAL in February 2020. We thank the Director, BSI and Head of Office, CNH for providing the necessary research facilities. We also thank Dr M. Sanjappa (former Director, BSI) and Dr M. F. Watson (Royal Botanic Garden Edinburgh), for providing useful information.

Received 14 August 2020; revised accepted 25 November 2020

doi: $10.18520 / \mathrm{cs} / \mathrm{v} 120 / \mathrm{i} 6 / 997-1006$ 